\title{
Tegola Tiered Mesh Network Testbed in Rural Scotland
}

\author{
Giacomo Bernardi \\ g.bernardi@sms.ed.ac.uk
}

\author{
Peter Buneman \\ opb@inf.ed.ac.uk \\ School of Informatics \\ The University of Edinburgh, UK
}

\author{
Mahesh K. Marina \\ mmarina@inf.ed.ac.uk
}

\begin{abstract}
Many rural and remote communities around the world see themselves on the wrong side of the digital divide. In particular, there is evidence to suggest that there is a growing digital divide between urban and rural areas in terms of broadband Internet access with people living in rural areas having fewer choices and pay higher prices for slower speeds. This is true even in developed countries. Motivated by the above observations, there has been an increasing interest in deploying and researching low cost rural wireless networks with active community participation. This paper presents an overview of our efforts in this direction in deploying a rural $\mathrm{WiFi}$ based long distance mesh network testbed in the Scottish Highlands and Islands. We highlight the unique aspects of our testbed that differentiate it from other existing rural wireless testbeds. We also outline some of the research issues that are currently being investigated in this project.
\end{abstract}

\section{Categories and Subject Descriptors}

C.2.1 [Computer-Communication Networks]: Network Architecture and Design-wireless communication

\section{General Terms}

Experimentation, Measurement, Performance, Reliability, Design

\section{Keywords}

Digital divide, Internet access, Rural and remote areas, Broadband wireless access, Low cost, 802.11, Mesh networks, Testbeds, Tiered architecture, Directional antennas, Network reliability, Over-water radio propagation, Self-powered masts, Diversity

\section{INTRODUCTION}

There is a growing recognition of the detrimental impact of the digital divide as evident from the Tunis Commitment of the United This work was supported in part by The University of Edinburgh
Development Trust.

Permission to make digital or hard copies of all or part of this work for personal or classroom use is granted without fee provided that copies are not made or distributed for profit or commercial advantage and that copies bear this notice and the full citation on the first page. To copy otherwise, to republish, to post on servers or to redistribute to lists, requires prior specific permission and/or a fee.

WiNS-DR'08, September 19, 2008, San Francisco, California, USA

Copyright 2008 ACM 978-1-60558-190-3/08/09 ...\$5.00.
Nations (UN) sponsored World Summit on the Information Society (WSIS) [1]. Efforts are underway around the world at all levels right from the grassroots to bridge this divide. While it is common to assume that digital divide mainly concerns the wide disparity in information access between developed and the developing nations, a similar problem exists even within developed countries between urban and rural areas though the latter problem seems relatively easier to address given the infrastructure and cultural acclimation to technology [2]. Without easy and affordable access to information and communications technologies (ICT) like in urban areas, communities such as those in rural Scotland are severely disadvantaged in several ways (e.g., children's access to educational resources, economic development opportunities), which may potentially lead to their eventual migration to urban areas.

Our focus in particular is on rural-urban divide in terms of Internet access, which is a small but crucial element of the larger digital divide. By all accounts, the rural areas lag behind their urban counterparts in broadband Internet access even in developed countries with people living in such areas having fewer choices and pay higher prices for slower speeds [3]. This view is also reiterated in a newly published OECD broadband report [4], which is telling given most of the member countries are considered developed. The root of the problem lies in the fact that rural areas have low user density and large distances between user clusters [5, 6], which makes it prohibitively expensive to deploy wired access technologies such as those seen in urban areas (e.g., DSL, cable, fibre) unless mandated and heavily subsidized by governments. That leaves wireless as the only viable technology approach in the foreseeable future and there seems to be widespread consensus on this. WiFi technology (based on the IEEE 802.11 standard) with its low cost commodity hardware and operation in the unlicensed spectrum lends itself as natural, readily available, low cost and easily deployable alternative. This is more so the case with the addition of mesh networking capabilities and high gain directional antennas (for enabling long distance links) to adapt from its original intended use as a technology for indoor wireless local area networks to work in outdoor scenarios over large areas. The recognition that blanket coverage and mobility support are not needed in rural areas also work in its favor. Therefore, not surprisingly, WiFi has become the de facto technology choice among researchers and communities seeking outdoor wireless connectivity $[7,8,9,10,11,12,13,14,15,16,17,18]$.

Tegola is a research project led by the University of Edinburgh in collaboration with the University of the Highlands and Islands (UHI) aimed at investigating ways to provide low cost, robust broadband wireless Internet access to remote and rural areas such as those in the Scottish Highlands and Islands. As a part of this project, we are deploying a testbed to serve as a realistic experimentation platform for characterization and evaluation studies in line with the 
project goals. The goal of this paper is to give an overview of our testbed with particular emphasis on its unique aspects. The testbed connects remote communities in the northwest of Scotland known for its harsh weather and mountainous terrain, a challenging environment for deploying and operating wireless networks, though typical of several other areas including remote areas of Scotland and the rest of the British Isles. The network is organized as a tiered mesh somewhat similar to other deployments (e.g., $[14,17])$. For reasons outlined above, we have chosen to implement the testbed using WiFi, though the technical issues highlighted such as radio propagation over water are somewhat orthogonal to the specific wireless technology used.

While our testbed is similar in terms of network organization and the underlying wireless technology to some other existing research testbeds and community deployments in outdoor rural settings, it is unique in two key respects. First, our environment necessitates radio propagation across long distances over (sea) water, which has a significant impact on magnitude and variability of the observed received signal strength characteristics because of multipath reflections off of changing water levels (including due to tidal patterns) and signal attenuation due to water absorption. Consequently, the channel and link characteristics measurements presented here are in sharp contrast to the conclusions made about rural long distance 802.11 links in the recent literature [12]. Second, self-powered wireless masts in our testbed deployed on mountains to get line of sight are powered by a combination of different sources (wind and solar). This is different from other existing deployments, which are situated in areas with tropical climates with plenty of solar radiation and rely solely on solar power. We find that exploiting diversity of energy sources can significantly cut down the cost and size of the power system (that includes power generators and batteries).

The remainder of this paper is structured as follows. Next section reviews related work. In Section 3, we give an overview of the testbed along with details of the hardware and software used. Section 4 discusses the link planning prior to deployment, measured link characteristics post deployment and our initial attempts to understand and improve link performance. Section 5 focuses on self-powering wireless masts that are located far away from gridconnected power supplies. We finally conclude in Section 6.

\section{RELATED WORK}

The related work broadly can be divided into two categories: urban and rural outdoor mesh networks targeting high and low user densities respectively. Several urban mesh networks in the form of municipal or community wireless networks $[9,10]$ as well as research testbeds $[7,8]$ have emerged recently as an attractive alternative for broadband Internet access with wider coverage with less expense and deployment ease. For the most part, these deployments are characterized by the use of omnidirectional antennas for communication over short distances and single or multiple radios per node.

More closely related to our work are the (WiFi-based) rural outdoor mesh network deployments. There exist a few research testbeds $[12,11,14,13]$ and relatively more number of community deployments $[15,16,17,18]$ in Africa, Latin America and India. Many of these deployments, especially in Africa, simply share a single Internet connection (e.g., VSAT satellite connections) among several users much like in a typical mesh network setting, albeit in rural terrains sometimes using planned long distance links with high gain directional antennas to get the necessary coverage. Few deployments, however, employ an overlay of long distance links as in our case to cover a larger number of users over a wider area $[14,17$, 12]. Some testbeds span both urban and rural areas as in $[14,12]$.
But none of the WiFi-based rural outdoor mesh testbeds we are aware of involve over-water links. While the presence of foliage has been reported (e.g., [14]), its impact is different and potentially lower compared to multipath reflections off of changing water levels (including due to tidal patterns) and signal attenuation due to water absorption. As a result, the link characteristics observed in our setting are in sharp contrast with characterizations presented in recent literature. For instance, Sheth et al. [12] conclude that rural long distance WiFi links exhibit negligible loss rates as they experience little or no external interference from other $\mathrm{WiFi} /$ nonWiFi sources, and because of the very low multipath interference and low delay spreads due to long, line of sight (LOS) links. As another difference, every other existing outdoor rural wireless deployment that requires self-powered masts relies on solar energy as those deployments are in tropical climate areas. Following the same approach is not meaningful in our setting and would result in an expensive and bulky powering solution given the very different climatic conditions.

\section{TEGOLA TESTBED}

The Tegola project is aimed at investigating ways to provide low cost and reliable broadband Internet connectivity to rural and remote communities such as those in the Scottish Highlands and Islands. In this section, we give an overview of the testbed that is being deployed to aid in our research in this project.

Area. Figure 1 provides an illustration of the testbed on a map. The testbed is situated in the northwest of Scotland connecting rural (mostly coastal) communities in Glenelg and Knoydart peninsulas to the Sleat peninsula on the Isle of Skye with long distance wireless links over water across the Sound of Sleat. Population in each of these communities is around 50-100, more or less evenly spread across all age groups. These communities though inhabited are quite remote. In fact, Knoydart peninsula is regarded as mainland Britain's most dramatic and unspoilt wilderness area[19]; there is no road access to Knoydart, it is only reachable by boat or a 2day hike! Before our project began, residents in these communities have no broadband in sight via the traditional means from the ISPs, and even the existing telephone and dial-up Internet connections tend to be unreliable in some locations. Climate in this region is maritime temperate and frequent, unpredictable changes are common as is the case in the rest of the British Isles. Weather can be quite harsh as it can often be wet and cloudy with strong winds and things can change quite rapidly to severe conditions, especially on the mountains. From network deployment and operation standpoint, this region is quite challenging given its inhospitable weather conditions and rugged mountainous terrain.

Architecture. The testbed is organized as a tiered mesh network with backhaul mesh tier with long distance wireless links acting as the top tier of the capacity distribution network connecting different target communities with the site linked to the wired Internet; backhaul nodes in turn distribute the available bandwidth to rooftop nodes in the individual communities and further down to the end-user client computers. Figure 1 shows the deployed (toptier) backhaul wireless network. Nodes marked $S$ and $I$ are on the Sleat peninsula in the Isle of Skye, nodes $B$ and $C$ on the Glenelg peninsula, and node $K$ on the Knoydart peninsula. Node $S$ located on the campus of Sabhal Mor Ostaig (Gaelic college on Skye) is linked to the wired Internet. We have used WiFi as the underlying wireless technology given its low cost and ready availability. Currently our testbed connects around 15 houses from widely dispersed coastal and remote communities living in the villages of Arnisdale and Corran (connected via nodes $B$ and $C$ respectively) and the west coast of Knoydart (connected via $K$ ). The houses in Arnis- 


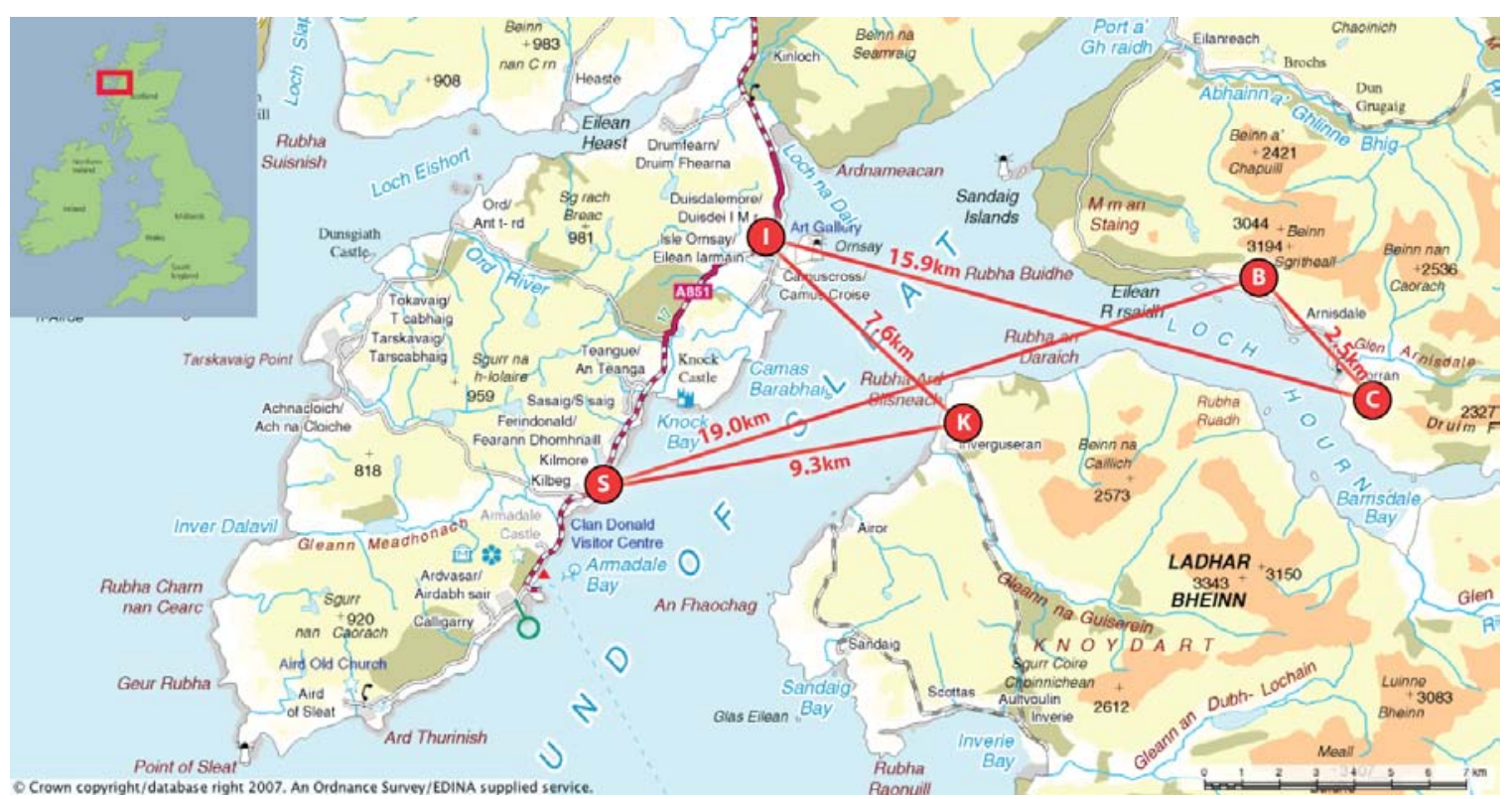

Figure 1: Tegola testbed on the map.

dale and Corran are linearly distributed along the coast, which is quite unlike that in typical urban mesh deployments.

Hardware. We use Avila GW2348-4 single board network processors from Gateworks as nodes in the backhaul tier shown in Figure 1. These boards, based on Intel IXP425 processor, come with $64 \mathrm{MB}$ RAM and 16MB Flash, and provide 4 mini-PCI slots and two Ethernet ports. In our configuration, one of the mini-PCI slots is typically used for local access via $802.11 \mathrm{~b} / \mathrm{g}$ in the $2.4 \mathrm{GHz}$ band, and the remaining 3 slots are used for backhaul connectivity over $802.11 \mathrm{a}$ in the $5 \mathrm{GHz}$ band. We have used mini-PCI radio modules from Ubiquiti Networks as they support higher transmit powers and exhibit better receive sensitivities - XtremeRange 5 (XR5) for $5 \mathrm{GHz}$ operation and XtremeRange2 (XR2) for $2.4 \mathrm{GHz}$. For the backhaul, we have used the high performance dish antennas operating in the $5 \mathrm{GHz}$ band with $29 \mathrm{dBi}$ gain and dual polarity support from Pacific Wireless; these antennas are quite rugged and also exhibit low cross-polarization effects. Each backhaul link is in fact comprised of two links using different polarizations (horizontal and vertical). This is useful for several reasons, including countering multipath fading and increasing the link capacity. We equipped the node $C$ with two Gateworks boards for added fault tolerance. For local access at each backhaul node, we are using 19dBi panel antennas from Pacific Wireless that operate in the $2.4 \mathrm{GHz}$ band. For access, we are using different types of hardware (Buffalo Wireless$\mathrm{G}$ router and access point, Ubiquiti NanoStations, alix3c2 boards from PC Engines) for rooftop mounted nodes. These nodes simultaneously act as station/client to the nearest backhaul node and as AP to devices within the house. We plan to experiment with the mesh mode in near future.

Nodes $B$ and $I$ in our testbed (see Figure 1) are self-powered using a wind generator as well as a solar panel. Node $B$ is in fact located on a mountain (called Beinn Sgritheall) at a height of about 1000 feet (see Figure 2 for a picture of this mast). We use the Rutland Furlmatic FM910-3 windcharger that can generate around $24 \mathrm{~W}$ at wind speeds around $5 \mathrm{~m} / \mathrm{s}$. We use the Kyocera KC130GH T-2 solar panel that has a maximum power output of $130 \mathrm{~W}$. To serve as a buffer during periods of low power generation, we use 2 identical Elecsol 125amp/hr 12v deep cycle batteries connected in

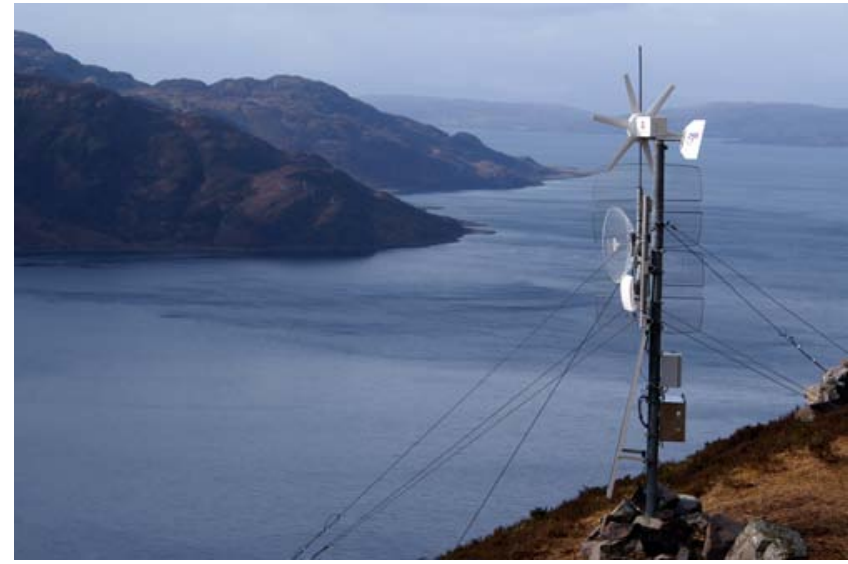

Figure 2: Self-powered mast on Beinn Sgritheall (node $B$ in Figure 1).

parallel and in turn connected to solar/wind power generators via a charge regulator. The load (Gateworks board) is connected to the battery bank on the other side.

Masts. Our masts need to be strong and rigid enough to withstand the substantial wind forces without deflection. They also need to be light enough that the individual components can be carried in by a small - three or four man - construction team. However they need not be tall: the terrain provides the height; the land is heavily grazed, so there is little chance of obstruction by vegetation; and the wind is strong enough that there is no need for height in order to obtain added wind velocity at the turbine. The main need for height is to keep the turbine clear of people and animals. Our initial design, shown in Figure 2, was a single, guyed, vertical pole supporting an " $\mathrm{H}$ " frame with approximately $2 \mathrm{~m}$ between the verticals to provide adequate separation of the dishes. However we found that we needed to install extra guy wires to stop the frame twisting.

Our current design uses aluminium scaffold poles and galvanized 


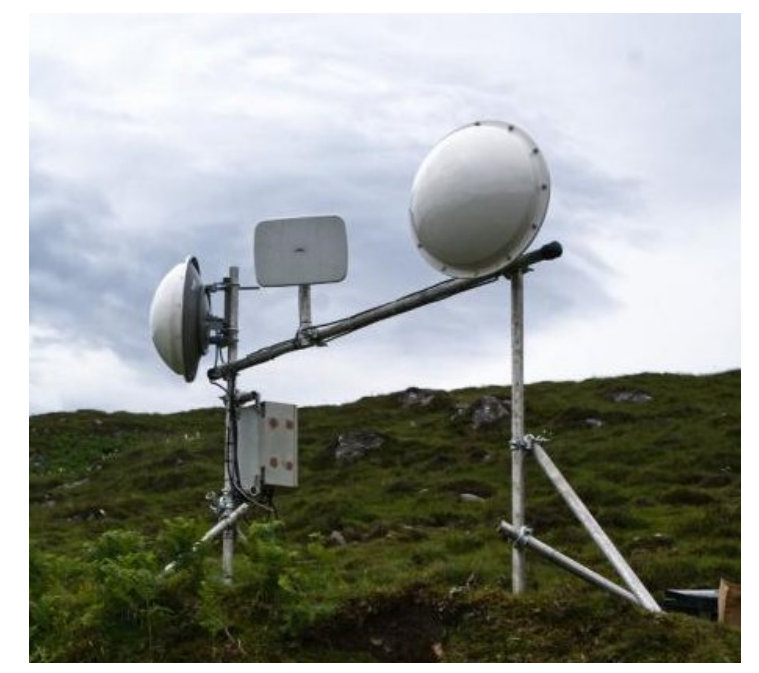

Figure 3: Mast at node $K$ in Figure 1, illustrating our current approach to mast construction.

connectors throughout. As shown in Figure 3, it consists of a horizontal bar at head height supported by two verticals about $2 \mathrm{~m}$ apart. The verticals support the larger (long-distance) antennae and the power generating equipment when present. The structure is diagonally braced with the same material. We have found this to be much better than using guy wires, which tend to become loose with time. The basic structure can be erected in a matter of minutes and is easy to adjust for uneven ground. A further improvement would be to incorporate some kind of shelter into the design — perhaps a tent using the horizontal bar as a ridge-pole. The Scottish Highlands seldom provides weather in which it is possible to do basic wiring, let alone electronics, outdoors.

It is also important to note that the materials required for building the masts were largely provided by the local communities. People from the communities also actively helped with the mast installations, which made it a significantly easier task.

Software. We have used OpenWrt with 2.6 Linux kernels as the operating system at all nodes. As radio driver, we used a slightly modified version of the MadWifi driver. Radios for the backhaul wireless links are configured to work in "adhoc demo mode" (pseudo IBSS) so that management frames nor beacons are ever sent in order to minimize the protocol overhead. RTS/CTS protection modes are disabled, and the ACK timeout and slot time values are configured using the MadWifi athcrtl utility based on the distance between the endpoints. We have used the default MadWifi rate adaptation algorithm, unless otherwise specified.

The entire network operates on a private IP address space, with OSPF routing in order to deal with route changes due to failure of any point-to-point link and for load balancing over multiple links (when using both polarizations simultaneously over different channels at the backhaul nodes). Connection to the Internet was via Linux server co-located at node $S$, which provides NAT and firewalling facilities.

In the UK, as per Ofcom regulations, high power transmissions for fixed wireless access requires a license and is only allowed in the $5725-5850 \mathrm{MHz}$ band with a maximum transmission power limit stipulated at $4 \mathrm{~W}$ EIRP. Therefore, we got a $5.8 \mathrm{GHz}$ fixed wireless access license and use only channels in that band in our testbed for backhaul communication.

\section{TOPOLOGY/LINK PLANNING AND CHARACTERISTICS}

Our goal in the initial phase of deployment was to connect the residents in the two villages of Arnisdale and Corran in the Glenelg peninsula and the communities in the west coast of Knoydart peninsula to the Internet (see Figure 1). Since connection to the Internet was only feasible via the Gaelic college on the Sleat peninsula in the Isle of Skye at location marked $S$ on the map, it was obvious that we needed long distance over-water radio links across the Sound of Sleat, but the exact mast locations and heights needed to realize this was not. We took a pragmatic stance in wanting to have a working system first before concentrating on the optimal mast placement problem, yet we wanted the topology to be redundant enough to survive a mast failure which naturally suggests seeking a "ring" topology to begin with. In fact, the decision to have redundancy in the backhaul has already proven useful. When one of the two boards at node $C$ failed, the network automatically switched to the alternate path without any noticeable disruption.

Our approach to identifying the suitable mast locations, though ad hoc, is straightforward and typical. We used topographic maps of the area in combination with the several GPS locations gathered via site surveying and also taking into account accessibility and closeness to a grid-connected power supply. Once we identified a pair of locations for a link, we tested for its feasibility using the Radio Mobile tool [20]. Two key observations came out of the above topology planning exercise: (i) only straightforward way to reach the Glenelg peninsula (the part of the map in Figure 1 with nodes $B$ and $C$ ) required a self-powered mast close to the top of a nearby mountain (Beinn Sgritheall); otherwise, we would have needed several (still self-powered) relays incurring a significantly higher cost. (ii) it was not possible to directly complete the "ring" from the Glenelg peninsula to Knoydart peninsula given that residents in Knoydart were along its west coast. This meant we had to bounce the signal off of a site on the Sleat peninsula in the Isle of Skye (the part of the map in Figure 1 with nodes $S$ and $I$ ). The resulting set of locations and links for the desired ring topology after testing the feasibility with Radio Mobile software are the ones shown in Figure 1.

In the rest of this section, we focus on our initial efforts to understand the impact of radio propagation over (sea) water on channel and link characteristics using the longest link in the testbed between nodes $S$ and $B$ (19Km long). We also evaluate the benefit of using a simple albeit inefficient remedy to overcome the signal degradation due to over-water propagation. We begin by looking at the feasibility test and link budget calculation for the link $S$ to $B$ using Radio Mobile software. Inputs to this tool are input transmit power, transmit and receive antenna gains, cable losses at sender and receiver, and receiver sensitivity at the desired rate. Given that we had radio hardware that supported high transmit power $(600 \mathrm{~mW})$ and antennas with high gain $(29 \mathrm{dBi})$, we had to lower one of them to comply with the regulations (4W EIRP limit). We chose to use a lower transmit power as using a high gain antenna at both ends will benefit the resulting link budget, so we used a transmit power of $10 \mathrm{dBm}(10 \mathrm{~mW})$ leaving the transmit/receive antenna gains at $29 \mathrm{dBi}$. We assumed cable losses to be a reasonable value of $3 \mathrm{~dB}$ at each end. Since we are interested in the feasibility of operating the link at the highest rate, we used the receive sensitivity of $74 \mathrm{dBm}$ corresponding to $54 \mathrm{Mbps}$ obtained from the XR5 card data sheet. Note that Radio Mobile automatically calculates the path loss using the supplied terrain data and relevant climate settings using Longley-Rice propagation model. With the above information, Radio Mobile predicted a received signal level of $-69.7 \mathrm{dBm}$, a link 




Figure 4: Radio Mobile output for the link $S$ to $B$ in the testbed with a transmit power of $10 \mathrm{dBm}$, transmit/receive antenna gains of $29 \mathrm{dBi}$ and $3 \mathrm{~dB}$ cable loss at each end.

margin around $4 \mathrm{~dB}$ which is somewhat lower than the typically desired margin of 10-20dB. The snapshot of the output from Radio Mobile is shown in Figure 4.

Our next step was to study the behavior of the measured receive signal strength value over time for this same $S$ to $B$ link along with the achievable link capacity seen at the higher protocol layers. For fine-grained sampling of the signal strength at the receiver, we used a light ping traffic between sender and receiver every $500 \mathrm{~ms}$. In addition, we used the widely used pathrate [21] every two minutes in the $S$ to $B$ direction to estimate the average link capacity. Measurement results are shown in Figure 5. Note that the light red lines in Figure 5 correspond to the receive sensitivity values at different rates as specified in the XR5 card data sheet. Looking at this data, we can make two main observations: (1) While the mean RSSI value from the measurements is close to the predicted value obtained from Radio Mobile, instantaneous RSSI value exhibits significant fluctuations by as much as $20 \mathrm{~dB}$ in a short span of 1-2 hour period. Such fluctuations are much higher than anything reported in the literature for rural long distance 802.11 links [14, 12]. We should mention here that we also monitored the signal strength variations for the other link between nodes $B$ and $C$ that is relatively shorter and over the land. While RSSI is much higher as expected, we also noticed that the variations are quite small within a $4 \mathrm{~dB}$ range and mostly within a $2 \mathrm{~dB}$ range. (2) Changes in average link capacity estimates are fairly well correlated with the signal strength variations with the capacity dropping by more than half during periods with low signal levels.

We suspect that the water level changes in the Sound of Sleat according to tidal patterns to be a major factor behind the large signal strength variations. In fact, tide heights can vary by as much as 7 meters in a six hour period. While a direct comparison of signal strength measurements with the tidal level variation data obtained for the same period does not suggest any correlation, a simple two ray reflection model to predict the received signal strength with varying tide levels with identical transmitter and receiver antenna heights as in the testbed does tend to confirm our hypothesis (see Figure 6). This model (details omitted for brevity) essentially determines the instantaneous difference in length between the direct path and the path of the reflected wave over sea water based on the tide level at that particular instant. Difference in path lengths in turn

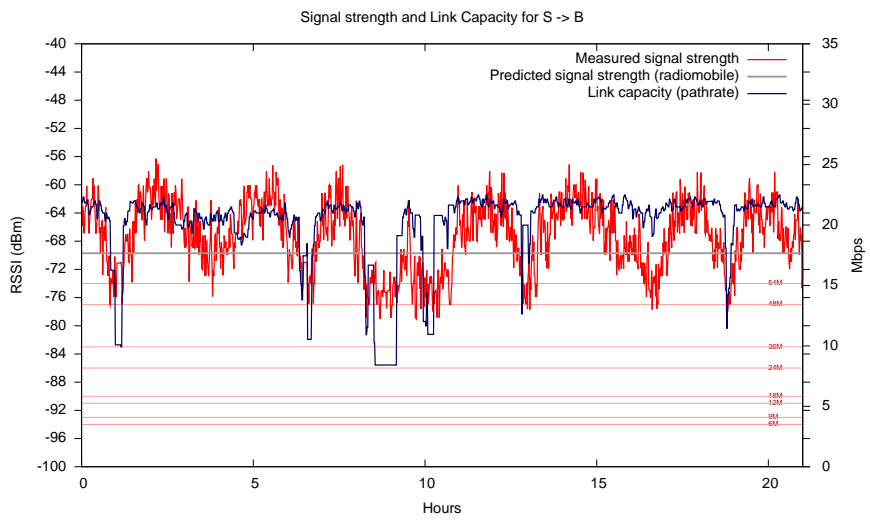

Figure 5: Measured signal strength and link capacity values for the link $S$ to $B$ over a 24 hour period.

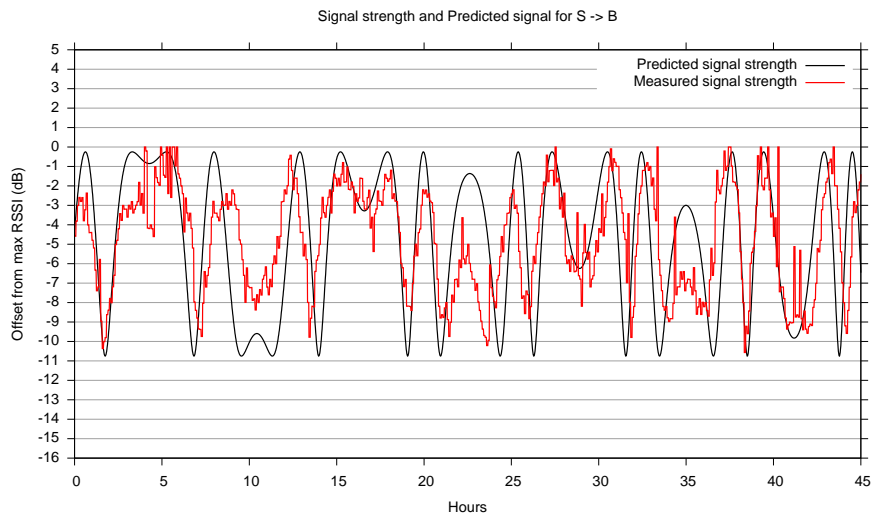

Figure 6: Comparison of measured signal strength values with the values predicted by a simple two-ray reflection model taking into account water level variations over a 45 hour period (almost two days).

leads to amplitude and phase changes of the receive signal level. We should mention, however, that other factors not accounted in the current model such as atmospheric pressure may also contribute to the RSSI variations.

The discussion so far clearly suggests that RSSI fluctuates significantly at the timescales of an hour, resulting in lowered effective link capacity and that it may be potentially because of the tide level variations. So the next natural question is to explore possible remedies to overcome such signal degradations. Diversity is a natural approach to explore and we evaluate a particular instance of spatial and antenna diversity. Specifically, we enable a second link between the two nodes $S$ and $B$ over a different channel and using the other polarization (vertical). Note that the results presented so far correspond to using the horizontal polarization and using one radio and antenna at each end. In the new setup, each end uses two radios, but still one antenna since it provides support for dual polarities. Figure 7 shows the results using this dual polarization approach corresponding to the same 24 hour period as in Figure 5. In this plot, the combined case is essentially picking the maximum signal strength between horizontal and vertical polarizations. Clearly, there is a noticeable and substantial (more than $5 \mathrm{~dB}$ gain) with this approach, which is promising but is not a very efficient solution since we are essentially doubling the resources 


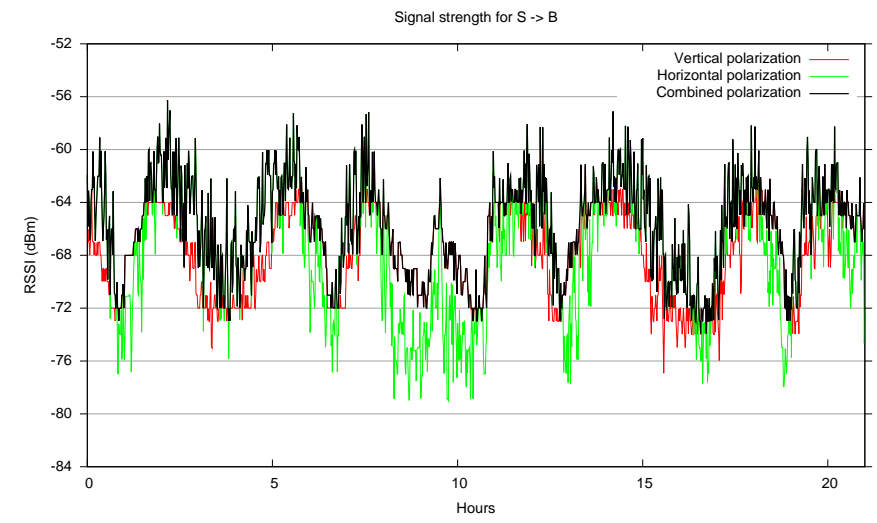

Figure 7: Benefit of using a simple dual polarization based diversity approach.

in terms of radios and channels. Our on-going work is exploring alternate and more efficient solution approaches.

\section{POWER PLANNING}

In this section, we look into the question of designing a low cost and small size powering system for self-powered masts. Recall that two backhaul nodes in our testbed (nodes $B$ and $I$ ) are mounted on self-powered masts. We use the mast at node $B$ as an illustrative example here to compare two approaches: (i) relying solely on solar power as done in every other deployment; (ii) combining different power sources (solar and wind).

To begin with, we start by looking at the power demand of the Gateworks board at node $B$. Based on the board's data sheet, it consumes $5 \mathrm{~W}$ without any radios and can take up to an additional $18 \mathrm{~W}$ to power the 4 miniPCI sockets, for a maximum power consumption of $23 \mathrm{~W}$. Since in fact, all four slots are used at the moment -2 802.11a cards for the dual polarization based approach outlined in the last section for the link between $S$ and $B, 1802.11$ a card for the link between $B$ and $C$ and $1802.11 \mathrm{~b} / \mathrm{g}$ card for local access at $B-$ the power consumption of the board is indeed $23 W$. For always-on operation, the total energy demand per day is $23 * 24=552$ Watt-hours $($ Wh $)$.

Suppose that with both approaches we want to ensure always-on operation and continue running up to $N$ "powerless" days. This would mean that in the case of solar-only approach, the mast will keep functioning for up to $N$ consecutive sunless days. The value of $N$ essentially determines the required amount of battery storage capacity. Given the above per day energy demand, the total energy demand over a $N$-day period is $552 * N W h$. Assuming the nominal voltage of the board is $12 \mathrm{VDC}$ (note that Gateworks board can support anywhere from $9-48 V D C$ ), we need $552 * N / 12 A h$ useful battery capacity $=46 * N A h$. With the two Elecsol $125 \mathrm{Ah}$ batteries we have operating at or below $80 \%$ depth of discharge (to ensure they last their full rated lifetime of 1000 deep discharge cycles), we get a useful battery capacity of $200 A h$ overall as opposed to the nominal capacity of $250 \mathrm{Ah}$. With $200 \mathrm{Ah}$ total battery capacity, the node $B$ system can continue to run for $N=200 / 46=4.35$ powerless days, which is a reasonably sufficient buffer.

Now consider the first approach of relying solely on solar power. Here we essentially want to determine the number of solar panels required to continuously power a $23 W$ load. But this in turns depends on the solar irradiation data for the worst month. Using the exact GPS coordinates of node $B$ mast location and the

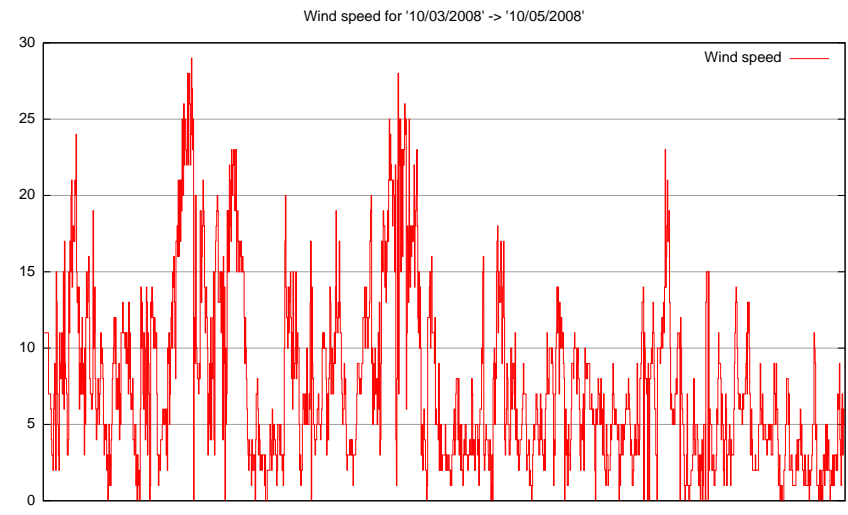

Figure 8: Measured wind speed at the nearest weather station for the period 10 March, 2008 to 10 May, 2008.

PVGIS solar irradiance data utility [22], we obtained the average irradiation $\left(W h / m^{2} / d a y\right)$ for each of the twelve months assuming panels are mounted at optimal inclination angle. The worst month for our mast site turns out to be December with an irradiation of $520 W h / \mathrm{m}^{2} /$ day. Using the same PVGIS utility, we have obtained the estimated energy production per month for the panel type we have (crystalline silicon with peak power output of $130 W$ ) to be $2 K W h$ over the whole month of December (the worst month). Thus to meet our energy demand of $552 \mathrm{Wh} /$ day (or 17.1 $K W h /$ month), we would need around 8 more such panels or a total of $9130 \mathrm{~W}$ panels. With each panel costing around $450 \mathrm{GBP}$, the total cost of the generation part of the system would be in excess of $4000 G B P^{1}$.

Let us now turn to the other approach of relying on a combination of energy sources. Specifically, let us consider wind as it is a plentiful resource in regions such as those of our testbed. Wind power generated depends on the wind speed. To get an estimate of the expected wind speed at our mast location, we used the BERR's wind speed database [23], which apparently is fairly accurate for rural areas. We have extrapolated the output from this database to account for the greater height of our mast location (which is $300 \mathrm{~m}$ ) to get an annual mean wind speed estimate of $9 \mathrm{~m} / \mathrm{s}$. This would result in 5 amps current generation to a $12 \mathrm{~V}$ battery with our Rutland Furlmatic 910-3 wind generator based on the specification data. We can expect about $48 \mathrm{~W}$ power generation (assuming $20 \%$ loss), which is more than sufficient by itself to power the Gateworks board. However, our calculations assume that wind speed remains constant throughout the year which is far from the truth. Looking at the measured wind speed data over a two month period at the nearest weather station (see Figure 8) shows considerable variability both within a day and across days, ranging from $0 \mathrm{~m} / \mathrm{s}$ to $30 \mathrm{~m} / \mathrm{s}$, with mean of $7.68 \mathrm{~m} / \mathrm{s}$. This data also suggests the need for greater battery storage capacity for absorbing the spikes in power generation. Given the above discussion, the question is whether we would additionally need solar power to continuously power our load. To answer this question, we use the historical wind speed and solar irradiation data obtained from a climate database [24] for a nearby place called Portree. This data is presented in Figure 9 and shows good agreement with the recent measured wind speeds from the nearest weather station. This data clearly shows that solar and wind

\footnotetext{
${ }^{1}$ This costing, however, does not include the components of the powering system common to both cases such as batteries and charge controller, which add a further $400 G B P$.
} 


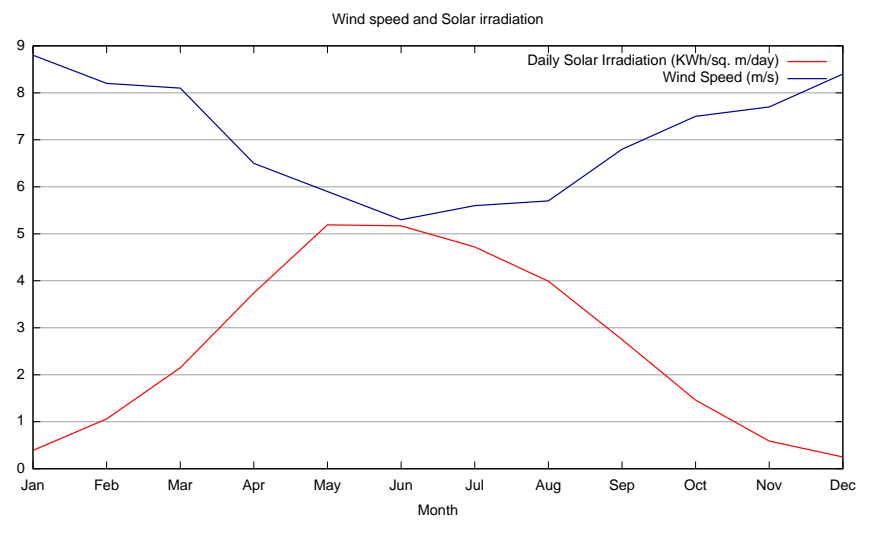

Figure 9: Historical monthly data for wind speed and solar irradiation data obtained from RETScreen climate database.

energy sources nicely complement each other, with wind power generation peaking in the winter months and solar power peaking in the summer months. Using data sheets for our wind generator and solar panel along with this data, we can in fact conclude that we can indeed meet our energy demand of about $0.5 \mathrm{KWh} /$ day throughout the year with just one solar panel and one wind generator. Given that both cost about the same, this gives a total cost of $900 G B P$ for the generation part of the powering system which is less than fourth of the cost compared to the previously discussed solar-only option. So the main message from the foregoing discussion is that exploiting the diversity of energy sources can significantly reduce the cost and size of the powering system in self-powered masts.

Even though we have given careful consideration to power planning for the self-powered masts in our testbed, the powering system is still the most expensive part of the hardware at such masts suggesting that there is motivation for further reducing the cost of the system. We plan to further explore this issue in future by looking at ways to reduce the power consumption requirements using intelligent power management techniques such as adaptive duty cycling. Also the above discussion is based primarily on historical and model data. While such data is quite useful to guide system size planning decisions, it can still deviate from the reality, so strictly following this data leaves room for the possibility of occasional power related outages. So we are in the process of adding power and weather monitoring capabilities to our self-powered masts. Finally, the network planning and power planning are interrelated issues, so need to be considered to be together. For instance, careful network planning can reduce the required node transmit power levels, thereby resulting in reduced power consumption and a cheaper powering system.

\section{CONCLUSIONS}

In this paper, we have described the Tegola tiered WiFi-based mesh network testbed being deployed in rural Scotland to enable research into low cost and robust broadband Internet connectivity to remote communities. This testbed differs from other existing rural outdoor mesh network deployments in two key aspects: (i) the presence of long distance over-water links; (ii) the need for relying on energy sources beyond solar to keep cost and size of selfpowered masts low. In addressing these issues, exploiting diversity emerged as a common theme in the solutions. Moreover, our design uses judicious amount of redundancy at various levels for uninterrupted and robust operation (e.g., backhaul ring topology, multiple router boards at the Internet gateway node, dual battery bank at self-powered masts). This design choice has proven to be quite useful with the network functioning almost uninterrupted over a 6month period by adapting to hardware failure at backhaul nodes and by being immune to variations in output from power sources at self-powered masts. Besides the two issues of over-water propagation and power planning discussed in this paper, we are looking into other related technical issues relevant to rural broadband wireless access, including automated network planning and management tools, QoS, and improving application robustness in such environments.

\section{REFERENCES}

[1] Tunis Commitment. http://www.itu.int/wsis/docs2/tunis/off/7.html, Nov 2005.

[2] B. Alfonsi. Bridging the Digital Divide. IEEE Pervasive Computing, Apr 2006.

[3] BBC News. Broadband Digital Divide Looms. http://news.bbc.co.uk/1/hi/technology/7115850.stm, Dec 2007.

[4] Organization for Economic Co-operation and Development (OECD). Broadband Growth and Policies in OECD Countries: Main Findings, 2008. Ministerial Background Report.

[5] E. Brewer. Technology Insights for Rural Connectivity. In Proc. Workshop on Wireless Communication and Development: A Global Perspective, Oct 2005.

[6] L. Subramanian et al. Rethinking Wireless in the Developing World. In Proc. ACM Workshop on Hot Topics in Networks (HotNets), 2006.

[7] J. Bicket, D. Aguayo, S. Biswas, and R. Morris. Architecture and Evaluation of an Unplanned 802.11b Mesh Network. In Proc. ACM MobiCom, 2005.

[8] J. Camp, J. Robinson, C. Steger, and E. Knightly. Measurement Driven Deployment of a Two-Tier Urban Mesh Access Network. In Proc. MobiSys, 2006.

[9] M. Mandviwalla et al. Muncipal Broadband Wireless Networks. Communications of the ACM (CACM), 51(2), Feb 2008.

[10] R. D. J. Kramer, A. Lopez, and A. M. J. Koonen. Muncipal Broadband Access Networks in the Netherlands: Three Successful Cases and How Europe May Benefit. In Proc. First International Conference on Access Networks (AccessNets), 2006.

[11] B. Raman and K. Chebrolu. Experiences in using WiFi for Rural Internet in India. IEEE Communications, 45(1), Jan 2007.

[12] A. Sheth et al. Packet Loss Characterization in WiFi-based Long Distance Networks. In Proc. IEEE Infocom, 2007.

[13] D. Wu, D. Gupta, and P. Mohapatra. Qual Ridge Wireless Mesh Network: Experiences, Challenges and Findings. In Proc. IEEE/Create-Net TridentCom, 2007.

[14] D. Gokhale, S. Sen, K. Chebrolu, and B. Raman. On the Feasibility of the Link Abstraction in (Rural) Mesh Networks. In Proc. IEEE Infocom, 2008.

[15] Wireless Networking in the Developing World. http://wndw.net/index.html, Dec 2007. Second Edition.

[16] Wray Village Community Network. www.wrayvillage.co.uk/wraycomcom.htm.

[17] K. W. Matthee, G. Mweemba, A. V. Pais, G. van Stam, and M. Rijken. Bringing Internet Connectivity to Rural Zambia Using a Colloborative Approach. In Proc. 2nd IEEE/ACM ICTD, 2007. 
[18] D. Johnson. Evaluation of a Single Radio Rural Mesh Network in South Africa. In Proc. 2nd IEEE/ACM ICTD, 2007.

[19] The Rough Guide to Scotland, Apr 2006. Seventh Edition.

[20] Radio Mobile radio system performance prediction tool. http://www.cplus.org/rmw/english1.html.

[21] Pathrate: A Measurement Tool for the Capacity of Network Paths.

http://www.cc.gatech.edu/fac/Constantinos.Dovrolis/pathrate.html.
[22] PVGIS Solar Irradiance Data Utility. http://sunbird.jrc.it/pvgis/.

[23] BERR Wind Speed Database. http://www.berr.gov.uk/energy/sources/renewables /explained/wind/windspeed-database/page27708.html.

[24] RETScreen Climate Database. http://www.retscreen.net/. 